\title{
Comunicação de notícias difíceis para pacientes sem possibilidade de cura e familiares: atuação do enfermeiro
}

\author{
Nurses' role in breaking difficult news to incurable patients and their relatives \\ Comunicación de noticias difíciles para pacientes sin posibilidad de cura y su familia: \\ actuación del enfermero
}

\author{
Cristiani Garrido de Andrade'; Solange Fátima Geraldo da Costa ${ }^{\text {IIl }}$ Maria Emília Limeira Lopes ${ }^{\text {III }}$ \\ Regina Célia de Oliveira ${ }^{I V}$; Maria Miriam Lima da Nóbrega ${ }^{V}$; Fátima Maria da Silva Abrãovi
}

\begin{abstract}
RESUMO: O estudo objetivou investigar a atuação do enfermeiro no que concerne à comunicação de notícias difíceis ao paciente sem possibilidade de cura e a aos seus familiares. Trata-se de uma pesquisa descritiva, de natureza qualitativa, na qual participaram 28 enfermeiros assistenciais atuantes em unidades de internação de um hospital público da cidade de João Pessoa - PB, no período de agosto a outubro de 2012. Para a coleta dos dados utilizou-se um formulário. Os dados obtidos foram tratados mediante a técnica de análise de conteúdo. Da análise do material empírico, emergiram duas categorias temáticas: Comunicação de notícias difíceis ao paciente e aos seus familiares: participação do enfermeiro; e Estratégias adotadas por enfermeiros para facilitar a comunicação de notícias difíceis ao paciente e aos seus familiares. Conclui-se que o enfermeiro assume um papel essencial na comunicação de más notícias ao paciente sem possibilidades terapêuticas de cura e à sua família. Palavras-Chave: Comunicação; paciente terminal; enfermagem; saúde.
\end{abstract}

ABSTRACT: The study investigated the nurses' role with regard to disclosing difficult news to incurable patients and their families. This qualitative, descriptive study involved 28 care nurses working on wards at a public hospital in João Pessoa, Paraíba, Brazil, from August to October 2012. A form was used to collect data, which was then analyzed by content analysis. Analysis of the empirical material revealed two thematic categories: giving difficult news to incurable patients and relatives: participation by nurses; and strategies used by nurses to facilitate the giving of difficult news to patients and relatives. It was concluded that nurses play an essential role in breaking bad news to patients with no therapeutic possibility of cure and their relatives. Keywords: Communication; terminally ill; nursing; health.

RESUMEN: El estudio objetivó investigar el papel de las enfermeras en relación a la comunicación de noticias difíciles a los pacientes sin posibilidades de cura y a sus familias. Se trata de un estudio descriptivo, cualitativo, en lo cual particparon 28 enfermeros que trabajan en las unidades de hospitalización de un hospital público, en la ciudad de João Pessoa - PB - Brasil, en el período de agosto a octubre de 2012. Para la recolección de datos se utilizó un formulario. Los datos fueron tratados mediante la técnica de análisis de contenido. Del análisis del material empírico, surgieron dos temas: La comunicación de malas noticias para los pacientes y sus famílias: participación del enfermeiro; y Estrategias adoptadas por enfermeros para facilitar la comunicación de noticias difíciles al paciente y a familias. Se concluye que el enfermero tiene un papel fundamental en la comunicación de malas noticias al paciente sin posibilidades terapéuticas de cura y a su familia.

Palabras Clave: Comunicación; enfermo terminal; enfermería; salud.

\section{INTRODUÇÃO}

Em um contexto de desenvolvimentos técnico e científico ímpares, na história das ciências da saúde, em que se acredita que quase todos os problemas podem ser determinados com o uso de aparatos tecnológicos, a terminalidade e a morte permanecem como limites para o ser humano. Dessa forma, pacientes, familiares e até mesmo os profissionais da área de saúde evitam falar sobre o tema ${ }^{1}$.

Cria-se, assim, uma circunstância conhecida como conspiração ou pacto do silêncio. Essa condição

${ }^{I}$ Enfermeira. Fonoaudióloga. Mestre em Enfermagem. Membro e Pesquisadora do Núcleo de Estudos e Pesquisas em Bioética. Docente em Enfermagem pela Faculdade de Ciências Médicas da Paraíba, Universidade Federal da Paraíba. João Pessoa. Paraíba, Brasil. E-mail: cristiani garrido@hotmail.com. IIEnfermeira. Doutora em Enfermagem pela Universidade de São Paulo. Coordenadora do Núcleo de Estudos e Pesquisas em Bioética. Universidade Federal da Paraíba. João Pessoa, Paraíba, Brasil.E-mail: solangefgc@gmail.com.

IIIEnfermeira. Doutora em Educação pela Universidade Federal do Rio Grande do Norte. Docente do Curso de Graduação e de Pós- Graduação em Enfermagem da Universidade Federal da Paraíba. João Pessoa, Paraíba, Brasil. E-mail: mlimeiralopes@yahoo.com.br.

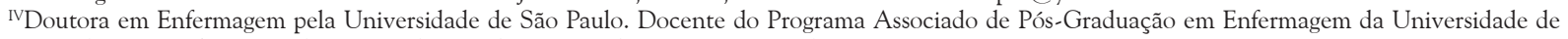
Pernambuco. Recife, Pernambuco, Brasil. E-mail: reginac_oliveira@terra.com.br.

vDoutora em Enfermagem. Docente do Programa de Pós-Graduação em Enfermagem da Universidade Federal da Paraíba. João Pessoa, Paraíba, Brasil. E-mail: miriamnobrega@uol.com.br.

v'Enfermeira. Doutora em Enfermagem pela Universidade de São Paulo. Coordenadora do Programa Associado de Pós-Graduação em Enfermagem da Universidade de Pernambuco e Universidade Estadual da Paraíba. Recife, Pernambuco, Brasil. E-mail: fatimabrao@terra.com.br. 
se manifesta com a transmissão de mensagens ambivalentes, em que o discurso verbal otimista e focalizado em assuntos variados e superficiais é contradito pela linguagem não verbal, que expressa de forma nítida a gravidade da situação ${ }^{2}$.

Pesquisas $^{3-5}$ destacam que receber informações honestas, claras e compassivas é um anseio universal dos pacientes com doenças em estágio avançado. Entretanto, eles também têm o direito de não querer sabê-lo. Assim, sua vontade deve ser respeitada, e a equipe pode identificar um familiar ou alguém próximo que receba uma má notícia e seja o interlocutor das mensagens ${ }^{2}$.

A notícia difícil ou más notícias é conceituada como qualquer informação que englobe mudança drástica na perspectiva de futuro da pessoa em um sentido negativo. É aquela que altera negativamente a expectativa do paciente em relação ao seu futuro, e sua resposta dependerá, entre outras coisas, de sua esperança de futuro, que é individual e influenciada por seu contexto psicossocial ${ }^{6}$.

É importante mencionar que a comunicação de notícias difíceis pode ser de três tipos: a comunicação do diagnóstico de doença avançada com prognóstico reservado; a comunicação e a atenção a graves sequelas dos tratamentos, tais como mutilação, prejuízo de funções e suas consequências na perda de qualidade de vida; a comunicação de esgotamento dos recursos de cura atual e a preparação para cuidados paliativos exclusivos $^{7,8}$.

Destaca-se que na passagem do tratamento com intenções de cura para a fase terminal, evidenciam-se as situações de difícil comunicação. Entre elas, contemplam-se as recidivas da doença pós-tratamento; as consequências de cirurgias mutiladoras e incapacitantes (para o trabalho, para a vida de relações, para a sexualidade, para a autonomia na vida diária); a toxidade e, várias vezes, a ineficácia dos tratamentos quimioterápicos e os efeitos adversos da radioterapia ${ }^{8}$.

Convém enfatizar que a comunicação do diagnóstico ao paciente é dever do médico e está prevista em seu código de ética profissional, porém a forma de fazê-lo deve ser de conhecimento de todos os profissionais da área de saúde, essencialmente do enfermeiro, uma vez que este permanece por mais tempo ao lado do leito do paciente. Além disso, a não comunicação só é consentida em casos de pacientes pediátricos ou quando suas condições físicas ou psicológicas não permitem que compreendam sua doença. Nesses acontecimentos, o diagnóstico deve ser comunicado à família ou ao responsável, porquanto é uma conduta de exceção, e o profissional deve saber reconhecer para quais pacientes a verdade deve ser omitida?.

Ressalta-se que, em geral, os enfermeiros ficam preocupados com o fato de o paciente/familiar conseguir suportar a escuta da informação sobre um diag- nóstico. Em outro momento, permanecem em conflito interno, entre contar, ou não, junto com o médico, a má notícia para o seu paciente e/ou seus familiares. Assim, precisam se questionar sobre a maneira como irão dividir essas informações com as pessoas envolvidas e informar-se sobre o que o paciente sabe, realmente, de seu diagnóstico e prognóstico ${ }^{10}$.

Diante do exposto, considerando a relevância da temática na área e o quantitativo ínfimo de estudos acerca da comunicação de más notícias aos pacientes sem possibilidade de cura, surgiu o interesse em desenvolver este estudo que teve como objetivo investigar a atuação do enfermeiro no que concerne à comunicação de notícias difíceis a esses pacientes e aos seus familiares.

\section{ReVisão de Literatura}

A preocupação com a comunicação de más notícias foi manifestada, desde a criação do primeiro código de ética médica dos Estados Unidos, em 1847, e, até a atualidade, o seu conteúdo e a sua forma são discutidos. Portanto, há debates constantes entre os profissionais da área de Saúde quanto à forma, ao tempo e ao momento adequado de dar a notícia do diagnóstico ao paciente e aos seus familiares, essencialmente o que se encontra sem possibilidades de cura. Assim, é uma ação delicada e difícil de ser administrada ${ }^{11}$.

A literatura esclarece que a comunicação de notícias difíceis é uma das mais penosas tarefas do profissional da saúde. Isso porque eles aprendem, nos bancos da academia, a salvar vidas e procurar a saúde, e não, a lidar com circunstâncias de perdas de saúde, vitalidade, esperança e morte ${ }^{2}$.

Os momentos de comunicação de notícias difíceis ocasionam perturbação, quer à pessoa que a recebe, quer à pessoa que a transmite, e provoca nos profissionais, principalmente nos enfermeiros, e nos pacientes medos, ansiedade, sentimentos de inutilidade, desconforto e desorientação. Esses aspectos conduzem, não raras vezes, a mecanismos de fuga nos profissionais, e devido ao medo de ser agredidos verbalmente, comunicam, comumente, de uma forma menos cuidada e menos simpática ${ }^{12}$.

Nesse contexto, são primordiais a percepção e a interpretação dos sinais não verbais do paciente, que consentirão a identificação do estado emocional do doente e mostrarão ao profissional até onde ir naquele momento. Ao comunicar notícias difíceis, é essencial que o profissional desponte atenção, empatia e carinho em seu comportamento e sinais não verbais. A expressão facial, o contato visual, a distância adequada e o toque em mãos, braços ou ombros auxiliam a evidenciar empatia e proporcionar apoio e conforto ${ }^{2}$. Nesse momento, a assistência do enfermeiro é primordial, uma vez que seu 
cuidado é embasado em uma atitude humanística, que contempla desvelo, solicitude, diligência, zelo, responsabilidade, preocupação e envolvimento com o paciente.

\section{Metodologia}

Trata-se de uma pesquisa descritiva, de natureza qualitativa. $\mathrm{O}$ cenário da investigação consistiu nas unidades de internação de um hospital público, localizado na cidade de João Pessoa - PB, considerado referência nesse Estado.

Os participantes da pesquisa foram 28 enfermeiros assistenciais da instituição selecionada para o estudo, que prestam cuidados direcionados ao paciente sem possibilidades de cura, selecionados da forma aleatória, mediante os seguintes critérios: que o profissional atuasse há pelo menos um ano na referida unidade; estivesse em atividade profissional, durante o período de coleta de dados; e com disponibilidade e interesse para participar da pesquisa, confirmando sua concordância com a assinatura do Termo de Consentimento Livre e Esclarecido.

A coleta de dados ocorreu, mediante entrevista, durante o período de agosto a outubro de 2012 e somente foi iniciada após a aprovação do projeto de pesquisa pelo Comitê de Ética em Pesquisa do Hospital Universitário Lauro Wanderley da Universidade Federal da Paraíba, conforme o CAAE no 02685412.2.0000.5183. Dessa forma, ressalte-se que o estudo foi realizado segundo a Resolução no 196/96¹3 do Conselho Nacional de Saúde, no que concerne às normas e às diretrizes regulamentadoras da pesquisa com seres humanos.

Para a obtenção do material empírico, foi utilizado um formulário com questões pertinentes aos objetivos propostos para a pesquisa: Você já teve a oportunidade de relatar más-notícias ao paciente na terminalidade e/ou aos seus familiares? Justifique. Como você acha que o profissional de enfermagem deve abordar as másnotícias ao paciente na terminalidade e aos seus familiares? Os enfermeiros foram contatados no hospital, e os formulários preenchidos em suas dependências, em local e hora apropriados para os profissionais.

Aplicou-se a técnica de saturação dos dados para limitar o contingente a ser pesquisado. A partir da 29a entrevista, as informações começaram a se repetir, determinando o encerramento da coleta de dados com 28 sujeitos.

Convém mencionar que, para manter o anonimato dos enfermeiros inseridos no estudo, os depoimentos oriundos das entrevistas foram identificados pela abreviatura Enf, seguida de números de 1 a 28 . Exemplo: Enf 2 e, assim, por diante.

Os dados obtidos, através dos instrumentos propostos, foram sistematizados qualitativamente, mediante a análise de conteúdo proposta por Bar$\operatorname{din}^{14}$, definida como conjunto de técnicas de análise das comunicações visando obter, por procedimentos sistemáticos e objetivos de descrição do conteúdo das mensagens, indicadores que permitam a inferência de conhecimentos relativos às condições de produção/ recepção destas mensagens.

Neste estudo, essa técnica realizou-se por meio dos seguintes passos: pré-análise, que consistiu em organizar os dados coletados por meio dos formulários; exploração do material, identificando-se os pontos relevantes de cada questão, com seus respectivos pontos convergentes, de acordo com o seu foco comum para, depois, agrupá-los em categorias e tratar os resultados, momento em que foram abordadas as inferências e as interpretações ${ }^{14}$.

Da análise, emergiram as seguintes categorias: Comunicação de notícias difíceis ao paciente e aos seus familiares: participação do enfermeiro; e Estratégias adotadas por enfermeiros para facilitar a comunicação de notícias difíceis ao paciente e aos seus familiares.

\section{Resultados e Discussão}

Neste estudo estão contempladas duas categorias temáticas cujos conteúdos desvelam como os enfermeiros utilizam a comunicação para abordar as más notícias ao paciente sem possibilidade de cura e a sua família.

\section{Comunicação de notícias difíceis ao paciente e aos seus familiares...}

No que diz respeito a essa categoria, os participantes resgatam que é dever do médico a informação de más notícias, como por exemplo, o diagnóstico de uma doença incurável, porém aludem que apesar de não ser do enfermeiro a responsabilidade da comunicação do diagnóstico, esse profissional assume um papel importante na integralidade do cuidado ao desenvolver estratégias que auxiliam o paciente a compreender a situação atual e aderir ao tratamento. As falas seguintes confirmam essa assertiva:

Geralmente essas notícias quem dão são os médicos, mas nós sempre acompanhamos os momentos seguintes a essa notícia, principalmente com a família. (Enf 18)

Aos poucos, junto com o apoio dos demais profissionais [psicólogos, médicos], vamos explicando as notícias ruins. (Enf 15)

[...] na consulta de enfermagem, eu acabo abordando a situação, todo o processo do tratamento, porque o médico informa, mas a pior parte fica para nós da equipe de enfermagem, que permanecemos por mais tempo com o paciente. (Enf 27)

A partir desses depoimentos, depreende-se que há uma responsabilidade maior do enfermeiro, no tocante ao acompanhamento do paciente e família após a comunicação de notícias difíceis, pelo fato de 
este profissional permanecer por mais tempo junto aos pacientes. Destarte, o fato de o enfermeiro ter mais contato com os pacientes no cotidiano do seu tratamento e de compartilhar dos sofrimentos, medos e angústias do paciente, ele tem condições de identificar e de se aproximar das necessidades sentidas e vividas pelos que estão sob sua responsabilidade, mais do que qualquer outro profissional. $\mathrm{O}$ vínculo estabelecido entre o enfermeiro e o paciente, no momento de uma notícia difícil, é essencial para ajudar o paciente a encarar sua nova realidade. É quando aflora a existência ou não do vínculo entre ambos ${ }^{9}$.

Assim, o papel que desempenha o enfermeiro na produção do cuidado inclui o dever de colaborar com o paciente e sua família, esclarecer-lhes e ter ciência de como e do momento em que deverá informar uma notícia difícil ${ }^{15}$. No que concerne a dar ou não uma notícia difícil, ressalte-se que, por achar que poderão aumentar o sofrimento e a depressão do paciente, alguns enfermeiros inseridos no estudo evitam falar sobre terminalidade e morte para preservar o paciente e sua família, visando proteger suas pessoas queridas; também fogem do assunto, como mostram os trechos a seguir:

Não acho que o paciente deve receber uma notícia ruim. O paciente já está passando por um momento difícil na sua vida, um momento de doença, no qual emerge sofrimento e dúvidas acerca da sua patologia, receber más notícias só iria aumentar tudo isso, piorando seu estado biopsicossocial e espiritual. (Enf 16)

Não sou a favor de transmitir uma má notícia, [...] existem determinados momentos que as más notícias provocam o agravamento da situação de vida do paciente. (Enf 17)

Se um paciente que não tem prognóstico, com seus dias contados, não tem porque estar fornecendo notícias ruins a ele, pois dependendo da notícia e da forma como ela é dada poderá acelerar o seu fim e proporcionar tristeza e/ou depressão. (Enf 28)

Os relatos evidenciados revelam que esses profissionais preferem ocultar a verdade a comunicar as notícias difíceis aos pacientes. Dessa forma, o estabelecimento do silêncio encobre as dificuldades do homem frente ao caráter inexorável da morte ${ }^{16}$. Intercalado com o silêncio, encontra-se a ocultação da verdade que, diversas vezes, acompanha uma informação de caráter mentiroso. Esse padrão de comunicação, sustentado pela sociedade, quando diante da proximidade da morte, parece ser uma forte característica cultural.

Um fato importante a se relatar é que, nos fins do Século XIX, o Código de Ética Médica dos EUA incentivava a ocultação da verdade ao paciente, revelando que é um dever sagrado guardar-se com cuidado a esse respeito, evitar todas as coisas que têm tendência a desencorajar o paciente e a deprimir seu espírito. Um século depois, a orientação é de que o médico deve lidar honestamente com o paciente e com seus colegas.
O paciente tem o direito de saber seu estado clínico, passado e presente, e de ficar livre de quaisquer crenças errôneas relativas à sua condição ${ }^{17}$. Assim, destaca-se a importância da revelação da verdade.

Vale salientar que, apesar das mudanças ocorridas, a crença generalizada de que a revelação ao paciente da incurabilidade de sua doença pode desencadear um processo depressivo e prejudicar a adesão ao tratamento ainda é uma forte justificativa para a ocultação da verdade:

[...] essas notícias podem levá-lo a depressão e/ou a doenças correlacionadas acelerando ainda mais a terminalidade. (Enf 18)

A omissão de informações e até a mentira podem acontecer na tentativa de minimizar o sofrimento do paciente e, possivelmente, dos profissionais e familiares, porém há consequências que interferirão na qualidade da relação profissional-paciente, principalmente, na forma como o paciente conduzirá seu tratamento e os momentos finais de sua vida ${ }^{16}$.

Atualmente, há uma preocupação constante quanto ao dever de informar, desde que a informação não provoque danos. Nesse caso, a comunicação deve ser feita ao seu responsável legal. Essas recomendações podem ser consideradas dúbias e gerar conflitos e inseguranças, se levar em conta a difícil identificação e previsão do que pode ou não causar danos psíquicos e emocionais ao paciente.

Alguns enfermeiros do estudo relataram tal fato, como evidenciam os trechos dos seguintes depoimentos:

[...] acho que devemos comunicar as notícias difíceis, dependendo de como o paciente apresenta o enfrentamento para este momento. (Enf 14)

[...] dependendo do estado dele, devemos comunicar, pois o paciente mesmo em fase terminal tem o direito de saber da verdade, [...] essa verdade dignifica sua pessoa como ser capaz e o insere no contexto de participação do cuidado. (Enf 13)

A notícia deve ser informada principalmente ao familiar de um paciente terminal que deve ter consciência da situação e da possibilidade de morte. (Enf 20)

Evidencia-se nestes depoimentos que os enfermeiros consideram necessária a comunicação de más notícias ao paciente e família. Uma pesquisa realizada no Brasil com 363 pacientes identificou que mais de 90\% dos entrevistados desejam ser informados sobre suas condições de saúde, incluindo eventuais diagnósticos de doenças graves ${ }^{4}$. Outro estudo europeu, efetivado com 128 indivíduos que tiveram o diagnóstico de câncer incurável, revelou que grande parte desses doentes gostaria de serem informados sobre opções de tratamento, efeitos colaterais, sintomas físicos e como e onde encontrar ajuda e aconselhamento a respeito de dietoterapia, cuidados psicossociais e complementares ${ }^{5}$. 
Em uma a revisão sistemática acerca das más notícias para pacientes com câncer, evidenciou-se que os pacientes mais jovens, do sexo feminino e os com maior nível escolaridade propagam maior desejo de receber informações mais detalhadas e concordam com a possibilidade de receber apoio emocional de um profissional. Nessa pesquisa, percebeu-se, ainda, que a influência de fatores demográficos interferiu na escolha de como receber a má notícia, como no caso dos asiáticos, que manifestaram mais interesse em receber a má notícia de modo presencial em detrimento dos ocidentais ${ }^{18}$.

É importante lembrar que, embora muitos pacientes queiram saber sobre sua condição de saúde, eles também têm o direito de não querer receber informações a esse respeito ${ }^{2}$. Assim, seu desejo carece ser respeitado. Por isso, é necessário identificar um familiar ou alguém próximo que possa receber as informações, para ser o interlocutor das mensagens.

Portanto, destaca-se que a comunicação de uma notícia difícil é uma prática comum para os profissionais de enfermagem que trabalham com pacientes sem possibilidades de cura, abrangendo a complexidade e a inter-relação do ser humano e de sua família, pelo fato de o momento da comunicação englobar a singularidade de cada ser.

\section{Estratégias adotadas por enfermeiros para facilitar a comunicação...}

Os discursos seguintes mencionam algumas estratégias utilizadas pelos enfermeiros para comunicarem más notícias ao paciente e/ou ao seu familiar:

Cabe ao profissional utilizar estratégias da comunicação e interação para saber qual o desejo do paciente e se este desejo for o de saber a verdade, deverá ter habilidade para fazê-lo (sabendo que esta é uma habilidade complexa) sem causar maiores danos psicológicos e físicos ao binômio paciente/familiar. (Enf 3)

De forma tranquila, segura, porém mostrando sentimentos acerca da situação que está sendo enfrentada. Mostrando-se solidário com o paciente e com a família, abraçando esse paciente. (Enf 1)

Inicialmente ouvir o paciente e a família, investigar o que os pacientes gostariam de saber, observar e perceber reações que lhes deem maior certeza sobre sua decisão de falar ou não naquele momento. (Enf 3)

Respeitando o momento certo, a hora adequada, o local correto, as palavras justas e sempre empaticamente. (Enf 13)

Esses depoimentos expressam que os enfermeiros consideram a comunicação de más notícias como uma tarefa complexa, que exige deles diversas habilidades, como, por exemplo: atenção, empatia e carinho, além de sinais não verbais. Acerca dessas habilidades, evidencia-se que a expressão facial, o contato visual, a distância adequada e o toque em mãos, braços ou om- bros auxiliam a demonstrar empatia e oferecer apoio e conforto ${ }^{2}$. Portanto, o paciente precisa sentir que, por pior que seja sua situação, ali se encontra alguém que não o abandonará, em quem poderá confiar e que poderá cuidar dele.

Para tanto, é importante o enfermeiro compreender o paciente, com uma expressão neutra e, posteriormente, informar as más notícias, de maneira clara e direta, utilizando um tom de voz suave, pausado e uma linguagem franca. Ele deve assegurar-se de que o paciente tenha compreendido a mensagem com clareza e emiti-la através de uma linguagem simples, com cautela, nos termos técnicos ${ }^{19}$.

Como estratégia para facilitar uma comunicação efetiva, destaca-se o protocolo de SPIKES, que estabelece táticas para uma comunicação eficiente quando for preciso dar más notícias, como: ter cuidado com o ambiente em que será emitido o diagnóstico; perceber as condições emocionais e cognitivas em que o paciente se encontra; ter uma conversa franca, sem ilusões e falsas expectativas; reconhecer as emoções e os sentimentos dos pacientes e sintetizar tudo o que foi dito ${ }^{20}$. Observa-se que algumas das táticas estabelecidas pelo citado protocolo foram referidas em alguns dos relatos dos participantes do estudo:

A abordagem deverá ser clara e objetiva para facilitar $o$ entendimento do paciente e familiar, respondendo suas indagações. (Enf 16)

Deve dizer a paciente apenas o que ele quer saber, tendo o cuidado para não ir além da sua compreensão. (Enf 11)

\section{[...] de forma planejada, gentil e cortês. (Enf 10)}

Os discursos dos enfermeiros apresentados nesse contexto demonstram que o emprego de tais táticas pode influenciar na comunicação das más notícias ao paciente. Ao utilizar as referidas técnicas, o enfermeiro passa a desenvolver habilidades na comunicação de modo a responder às indagações do paciente sem, no entanto, transcender a sua compreensão.

Nesse prisma, ressalta-se que tais habilidades comunicacionais fazem parte da profissão dos enfermeiros, razão por que seus currículos profissionais deveriam prepará-los para ela ${ }^{21}$. Desse modo, tornase necessária a inclusão formal de vivências durante a graduação que recorram a outras estratégias de ensino, como, por exemplo, o emprego de técnicas psicodramáticas utilizando-se a comunicação de más notícias $^{22}$. Esse fato foi referido:

É sempre complicado porque na graduação não somos preparados para esse tipo de abordagem. (Enf 18)

É importante ressaltar que comunicar notícias difíceis não é um assunto exclusivo dos médicos, mas também dos enfermeiros. Destarte, ao revelar o diagnóstico, tais profissionais não podem esquecer que 
estão diante de um paciente ou familiar que experimenta o impacto de saber que está com uma doença ameaçadora da vida ${ }^{23}$. Assim, destaca-se a importância de se avançar nos estudos acerca do processo de comunicação das más notícias, principalmente aos pacientes sem possibilidade de cura.

\section{CONClusão}

O presente estudo, por meio do qual se abordou a comunicação de notícias difíceis entre enfermeiros e paciente terminal, ressalta que esta tarefa é difícil de ser realizada, devido à falta de preparo para lidar com os aspectos subjetivos que envolvem esse processo, como o sofrimento manifestado pelo profissional e as reações do paciente.

Ficou evidente, através dos depoimentos dos participantes do estudo, que os enfermeiros assumem papel fundamental na integralidade do cuidado, ao desenvolver estratégias que ajudam o paciente a compreender sua situação atual e a aderir ao tratamento, promovendo um relacionamento interpessoal efetivo.

Portanto, o estudo colaborou no avanço do conhecimento da área de enfermagem, visto que evidenciou a relevância da comunicação de notícias difíceis de forma eficaz, como uma estratégia fundamental para respaldar a prática clínica do enfermeiro, direcionada ao paciente sem possibilidades de cura.

\section{REFERÊNCIAS}

1.Kovács MJ. Comunicação em cuidados paliativos. In: Pimenta CAM, Mota DDCF, Cruz DALM. Dor e cuidados paliativos: enfermagem, medicina e psicologia. Barueri (SP): Manole; 2006. p. 86-102.

2.Silva MJP, Araújo MMT. Comunicação em cuidados paliativos. In: Carvalho RT, Parsons HA. Manual de cuidados paliativos ANCP. 2 ${ }^{\text {a }}$ ed. Porto Alegre (RS): Sulina; 2012. p.75-85.

3.Aspinal F, Hughes R, Dunckley M, Addington-Hall J. What is important to measure in the last months and weeks of life?: a modified nominal group study. Int J Nurs Stud. 2006; 43: 393-403.

4.Gulinelli A, Aisawa RK, Konno SN, Morinaga CV, Costardi WL, Antonio RO, et al. Desejo de informação e participação nas decisões terapêuticas em caso de doenças graves em pacientes atendidos em um hospital universitário. AMB Rev Assoc Med Bras. 2004; 50(1): 41-7.

5.Voogt E, Van Leeuwen AF, Visser AP, Van der Heide A, Van der Maas PJ. Information needs of patients with incurable cancer. Support Care Cancer. 2005; 13: 943-8. 6.Mochel EG, Perdigão ELL, Cavalcanti MB, Gurgel WB. Os profissionais de saúde e a má notícia: estudo sobre a percepção da má notícia na ótica dos profissionais de saúde em São Luís/MA. Cad Pesq. 2010; 17(3): 47-56.
7.Jaccobsen J, Jackson VA. A communication approach for oncologists: understanding patient coping and communicating about bad news, palliative care, and hospice. J Natl Compr Canc Netw. 2009; 7: 475-80.

8.Ministério da Saúde (Br). Instituto Nacional de Câncer. Comunicação de notícias difíceis: compartilhando desafios na atenção à saúde. Rio de Janeiro: INCA; 2010.

9.Gomes CHR, Silva PV, Mota FF. Comunicação do diagnóstico de câncer: análise do comportamento médico. Rev Bras Canc. 2009; 55: 139-43.

10.Ordahi LFB, Padilha MICS, Souza LNAS. Comunicação entre a enfermagem e os clientes impossibilitados de comunicação verbal. Rev Latino-Am Enfermagem. 2007; 15(5): 85-93.

11.Pinheiro EM, Balbino FS, Balieiro MMFG, Domenico EBL, Avena MJ. Percepções da família do recém-nascido hospitalizado sobre a comunicação de más notícias. Rev Gaucha Enferm. 2009; 30(1): 77-84.

12.Pereira MAG. Má noticia em saúde: um olhar sobre as representações dos profissionais de saúde e cidadãos. Texto contexto - enferm. 2005; 14(1): 33-7.

13.Ministério da Saúde (Br). Resolução n ${ }^{\circ}$ 196, de 10 de outubro de 1996: aprova as diretrizes e normas regulamentadoras de pesquisa envolvendo seres humanos. Brasília (DF): Ministério da Saúde; 1996.

14.Bardin L. Análise de conteúdo. São Paulo: Edições 70; 2011.

15.Silva VCE, Zago MMF. A revelação do diagnóstico de câncer para profissionais e pacientes. Rev Bras Enferm. 2005; 58: 476-80.

16.Geovanini FCM. Notícias que (des) enganam: o impacto da revelação do diagnóstico e as implicações éticas na comunicação de más notícias para pacientes oncológicos [dissertação de mestrado]. Rio de Janeiro: Escola Nacional de Saúde Pública; 2011.

17.Franco F. Humanização na saúde: uma questão de comunicação. In: Epstein I, organizador. A comunicação também cura na relação médico e paciente. São Paulo: Angellara; 2006. p. 149-63.

18.Fujimori M, Uchitomi Y. Preferences of cancer patients regarding communication of bad news: a systematic literature review. Jpn J Clin Oncol. 2009; 39: 201-16.

19.Silva NH, Neman FA. Como comunicar más notícias. Science in Health. 2010; 1(3): 11-20.

20.Rosenzweig, MQ. Breaking bad news: a guide for effective and empathetic communication. NP News. 2012; 37(2): 1-4.

21.Tapajós R. A comunicação de notícias ruins e a pragmática da comunicação humana: o uso do cinema em atividades de ensino. Interface (Botucatu). 2007; 11(21): 165-72.

22.Ramos-Cerqueira ATA, Lima MCP, Torres AR, Reis JRT, Fonseca NMV. Era uma vez...contos de fada e psicodrama auxiliando alunos na conclusão do curso médico. Interface Comum Saúde Educ. 2005; 9(16): 81-9.

23.Gomes GC, Xavier DM, Mota MS, Salvador MS, Silveira RS, Barlem ELD. Dando notícia difíceis à família da criança em situação grave ou em processo de terminalidade. Rev enferm UERJ. 2014; 22: 347-52. 